\title{
Preface to selected papers from the 6th International Conference on Computational Systems-Biology and Bioinformatics (CSBio2015)
}

The rapid advancement in high-throughput technologies has shifted life science research into a "big data" paradigm. To unlock the full potential of the vast amount of data and translate it into benefits for society (e.g. better medicine and healthcare), novel and advanced computational tools are needed for data analysis. This issue is a follow-up to the 6th International Conference on Computational Systems-Biology and Bioinformatics (CSBio2015) that was held in Bangkok, Thailand, during 22-25 November 2015. The conference was co-organized by King Mongkut's University of Technology Thonburi (KMUTT), Kasetsart University (KU), and the National Center for Genetic Engineering and Biotechnology (BIOTEC). Notably for KMUTT, this event was a celebration of many milestones including the 55th anniversary of the university, the 20th anniversary of the School of Information Technology, and the 12th anniversary of her Bioinformatics and Systems Biology Program. More than 100 participants from 19 different countries attended the conference, making it truly international in scope. Among the 84 submissions received, the program committee members recommended the top-quality papers for this special issue. After a rigorous peer-review process, three papers were selected as outlined below.

The first paper, by Lu and Leong, entitled "GI-SVM: A sensitive method for predicting genomic islands based on unannotated sequence of a single genome" ${ }^{1}$ presents an approach based on one-class support vector machine (SVM) to identify bacterial genomic islands without relying on gene annotation information or comparisons with other closely related genomes. This makes it possible to quickly scan for genomic islands on newly unannotated genome sequences. Comprehensive performance evaluations and comparisons with other well-known methods were carried out and presented.

The second paper, by $\mathrm{Yu}$ et al., entitled " $\mathrm{An} \mathrm{O}\left(\mathrm{n}^{3}\right)$ algorithm for sorting signed genomes by reversals, transpositions, transreversals, and block-interchanges" ${ }^{2}$ describes a method for finding the fewest reversals, transpositions, transreversals, and block-interchanges to convert one genome into another assuming that they 
involve the same set of genes. The authors introduce a graph and a concept of parity and use these to define the bridge structures, which resulted in a significant improvement in terms of time complexity.

The third paper, by Behaegel et al., entitled "A hybrid model of cell cycle in mammals" 3 describes an approach for modeling gene regulatory networks by extending Rene Thomas's discrete framework to account for continuous times for state transitions. The authors applied their method to the mammalian cell-cycle system as a representative case and also showed how biological knowledge of the system can be used to constrain the parameters of the model.

We would like to take this opportunity to acknowledge all the authors who submitted papers to this special issue. We are also grateful to all the reviewers for their critical reviews that helped improve the quality of the papers. Finally, we would like to thank the organizers, committee members and supporting staffs of CSBio2015 for their efforts in making this event a successful one.

Weerayuth Kittichotirat King Mongkut's University of Technology Thonburi, Thailand

Worrawat Engchuan King Mongkut's University of Technology Thonburi, Thailand Wanwipa Vongsangnak Kasetsart University, Thailand

Asawin Meechai King Mongkut's University of Technology Thonburi, Thailand

\section{References}

1. Lu B, Leong HW, GI-SVM: A sensitive method for predicting genomic islands based on unannotated sequence of a single genome, J Bioinform Comput Biol 14(1):1640003, 2016.

2. $\mathrm{Yu} \mathrm{S}$ et al., $\mathrm{An} \mathrm{O}\left(\mathrm{n}^{3}\right)$ algorithm for sorting signed genomes by reversals, transpositions, transreversals, and block-interchanges, J Bioinform Comput Biol 14(1):1640002, 2016.

3. Behaegel J et al., A hybrid model of cell cycle in mammals, J Bioinform Comput Biol 14(1):1640001, 2016. 